\title{
Clinical Utility of Radiation Medicine 99mTc-Methylene Di- phosphanate Bone Scintigraphy in the Early Diagnosis of Rheumatoid Arthritis.
}

\author{
Karan Peepre ${ }^{1}$, Deepak Shah ${ }^{2}$, A.N. Tiwari ${ }^{1}$, R. Pisal ${ }^{1}$ \\ ${ }^{1}$ Institute: Nuclear Medicine division, Department of Radiation oncology, Gandhi Medical \\ College,Bhopal,India. \\ ${ }^{2}$ Consultant Orthopedic Surgry,Bhopal,India
}

\begin{abstract}
The value of 99mTc-Methylene diphosphanate (99mTc-MDP) bone scan in predicting development of bony destruction, swelling, new bone erosions and skeletal deformities in small joints and bones was studied by visual evaluation of bone scans. ${ }^{99 m} T c-M D P$ was injected intravenously. 3 hours after the $99 m T c-M D P$ injection, they have been underwent spot views of entire skeletons with planar scintigraphy in the anterior and posterior positions. All patients were examined under the Electronics Corporation of India Limited (ECIL) single head gamma camera imaging machine, equipped with a low-energy high-resolution parallel-hole collimator. In 9 patients with newly diagnosed rheumatoid arthritis a total of 60 patients of 99mTc-MDP bone scan were examined clinically, scintigraphically, and radiographically. The follow up period was 2 years. Seven patients found increased radiopharmaceutical increased in small joints. Onset of the rheumatoid disease was found in three out of seven patients. 2/9 patients, the joints which were eroded scintigraphically, was active at all the checkups. Erosions were detected earlier in foot joints than in finger joints. New erosions were especially prone to appear in joints with persisting and high scintigraphic activity. On the contrary, inactive joints by radionuclide scanning never eroded. Scintigraphic and clinical activity and radiographic erosion correlated significantly with each other. The sensitivity and specificity of visual scintigraphic assessment and the relative pixel activity method proved to be superior to the region of interest methods and clinical evaluation for prediction of bony erosions in patients of rheumatoid arthritis.
\end{abstract}

Key words: Bone scan, 99mTc-Methylene di-phosphonate, Gamma camera, Small joints, Bone erosion.

\section{Introduction:}

Rheumatoid arthritis (RA) is a form of inflammatory arthritis and an autoimmune disease of the joints. In RA, the immune system, which is designed to protect our health by attacking foreign cells such as viruses and bacteria - instead attacks the body's own tissues, specifically the synovium (synovial), a thin membrane that lines the joints. Rheumatoid arthritis is symmetrical, meaning if a joint on one side of the body is affected; the corresponding joint on the other side of the body is also involved. Because the inflammation is systemic, patients are likely to feel fatigued and may become anemic, anorexia and run a low-grade fever. Due to inflammatory attack, fluid builds up in the joints, causing pain in the joints and inflammation, which occur throughout the body. Patient's joints may feel warm to the touch and you might notice a decreased range of motion, as well as inflammation, swelling and pain in the areas around the affected joints. Most people with RA experience intermittent bouts of intense disease activity, called flares. In some people the disease is continuously active and gets worse over time. Others enjoy long periods of remission, no disease activity or symptoms at all. More than 50 million American adults (one in every five) have diagnosed arthritis. Arthritis affects 300,000 children under the age of 18.Two-thirds of people with arthritis are younger than 65 years old. People of all genders, ages and races can get arthritis. However, women are more likely to get it than men. RA causes work limitations; there are 2.5 times as many women as there are men with rheumatoid arthritis (RA).Arthritis prevalence increases with age, 50 percent of adults age 65 and older have a form of arthritis. Evidence shows that early diagnosis and aggressive treatment to put the disease into remission is the best means of avoiding joint destruction, organ damage and disability [1]. RA is generally worse in the morning and associated with stiffness; in the early stages, patients often have no symptoms after a morning shower. RA is a systemic inflammatory disease characterized by polyarthritis. Joint inflammation typically affects small peripheral joints, where bone erosions are also first seen [2]. Erosions of bone are the most important criterion of progressive arthritis and an indication for prompt institution of second line anti- rheumatic drug treatment [3]. Because clinical judgment is often inaccurate, objective methods are urgently needed to predict erosiveness of RA. Joint scintigraphy is an objective method of demonstrating and assessing joint inflammation [4-5]. Accumulation of bone seeking radioisotopes $99 \mathrm{mTc}-$ Methylene di-phosphonate reflects metabolic activity of 
bone and blood pooling in the joint. Skeletal scintigraphy with gamma camera is a more selective method for the detection of joint inflammation than radiography and more sensitive than clinical evaluation.

\section{Methods:}

A total of 9 patients were accounted for the radionuclide study. This study was performed at the division of Nuclear Medicine division, Department of Radiation Oncology, Hamidia \& Kamla Nehru Hospital, Gandhi Medical College, Bhopal,India. All patients was referred to nuclear medicine for bone scan with the complaints of low grade fever, swelling and pain in small joints. Patients were referred before treatment and during the follow-up. A dose of 20 to $25 \mathrm{mCi}$ of ${ }^{99 \mathrm{~m}} \mathrm{Tc}-\mathrm{MDP}$ was injected intravenously. 3 hours after the 99mTc-MDP injection, they have been underwent spot views of entire skeletons with planar scintigraphy in the anterior and posterior positions. All patients were examined under the Electronics Corporation of India Limited (ECIL) single head gamma camera imaging machine, equipped with a low-energy high-resolution parallel-hole collimator. All spot views were taken as the primary method of acquisition, the regions of the skeleton covered by each spot view. The first spot view of the axial skeleton, usually the posterior projection of the chest, was acquired for approximately 500,000 to 1 million counts depending on the field of view (FOV) of the gamma camera. Spot images were obtained using a 128.128 or a $256 \times 256$ matrix ( $>200,000$ counts).

Reporting: Nuclear medicine physician record appropriate information regarding the patient, especially type of examination, date, radiopharmaceutical (administered activity and route), a summary of patient history, all correlated data from previous diagnostic studies and the clinical problem. Findings, abnormal tracer uptake (increased, decreased, pattern of abnormal uptake, bone findings, soft tissue findings) were noted. We also wrote comparative data (correlation with other diagnostic results and comparison with previous studies in the reports. In Interpretation, a clear diagnosis is given.

\section{Discussions:}

In this prospective study, the radiological features characteristic of osteoarthritis of the hand were compared with the radionuclide bone scan images. A total of 32 patients were assessed at 6-monthly intervals for 18 months. Microfocal radiographs were taken at each visit. The high magnification and resolution of this technique permitted direct measurement of joint space width, subchondral sclerosis, osteophyte number and area and juxta-articular radiolucency area for each joint in the hand. Four-hour technetium 99m methylene diphosphonate bone scans were taken at 0 and 12 months and the activity of tracer uptake at each joint scored. The latter was compared with each X-radiographic feature at every visit and the changes between visits analysed. The scan scores did not correlate with any of the X-radiographic features other than osteophyte size. During the study the size of growing and remodelling osteophytes increased significantly at joints with raised or increased isotope uptake [6]. In our research study we found 7 out of 9 patients were positive bone scans with involvement of small joints and results were very encouraging. Several chemical and immunological markers have been claimed to predict disease progression or development of erosions in RA. These include rheumatoid factor [7-8] erythrocyte sedimentation rate, [9] anemia, [10] serum C reactive protein [11] serum Clq level, [12] and circulating immune complexes [13]. 99mTc-MDP bone scintigraphy for detecting rheumatoid arthritis is a very sensitive and specific radionuclide procedure used for early diagnosis. It is safe, cheaper and non invasive, and it can take scan of the entire skeleton along with spot views. It is a gold standard radionuclide procedure clinical useful in diagnosis of rheumatoid arthritis earlier then X-ray (radiograph) [14].

RA may affect many different joints and cause damage to cartilage, tendons and ligaments (long term effects). One common outcome is joint deformity and disability. Some people with RA develop rheumatoid nodules; lumps of tissue that form under the skin, often over bony areas exposed to pressure. These occur most often around the elbows but can be found elsewhere on the body, such as on the fingers, over the spine or on the heels. Over time, the inflammation that characterizes RA can also affect numerous organs and internal systems. Our study shows very encouraging results. Therefore, we recommend non invasive, cheap and sensitive method, 99mTc-MDP bone scan for early diagnosis of RA in patients to prevent joint/bony deformities and to provide good quality of healthy life.

\section{Results:}

This study show, in 9/60 patients with newly diagnosed rheumatoid arthritis of 99mTc-MDP bone scan were examined clinically, scintigraphically, and radiographically. The follow up period was 2 years. 7/9 patients found increased radiopharmaceutical increased in small joints. Onset of the rheumatoid disease was found in three out of seven patients. $2 / 9$ patients, the joints which were eroded scintigraphically, was active at all the examinations. Erosions were detected earlier in foot joints than in finger joints. New erosions were especially prone to appear in joints with persisting and 
high scintigraphic activity. On the contrary, inactive joints by radionuclide scanning never eroded. Scintigraphic and clinical activity and radiographic erosion correlated significantly with each other. The sensitivity and specificity of visual scintigraphic assessment and the relative pixel activity method proved to be superior to the region of interest methods and clinical evaluation for prediction of bony erosions in patients of rheumatoid arthritis.

\section{Conclusions:}

99mTc-MDP bone scan is very sensitive, specific and superior to conventional modalities to diagnose rheumatoid arthritis (RA) very early. The value of 99mTC-MDP scintigraphy in predicting development of new erosions in small peripheral joints was studied by visual evaluation of bone scan. Erosions can be detected earlier in foot joints than in finger joints. New erosions were especially prone to appear in joints with higher tracer uptake. The sensitivity and specificity of visual scintigraphic assessment proved to be superior to the region of interest methods and clinical evaluation for prediction of joint involvement and erosions of the bones.

\section{Acknowledgements:}

I would like to express my deepest appreciation to all the Orthopedic surgeons and consultants of Medicine of the city of Bhopal, India for referring patients for 99mTc-MDP scintigraphy. In addition, my sincere thanks to Professor Dr. Jagdish Prasad Sharma, Radiation safety officer, Dr.Mukul Mathur, who has shown the attitude and the substance of a genius, and continually supporting in regard to research, and an excitement in regard to study. Without their supports and constant help this research would not have been possible. I also thank to staff of the department of radiation oncology, Gandhi medical college, Bhopal, India for their cooperation and all assistance.

\section{References:}

[1] Rheumatoid Arthritis.Arthritis Foundation.

[2] Brook A, Corbett M. Radiographic changes in early rheumatoid disease. Ann Rheum Dis 1977; 36: 71-3.

[3] Luukkainen R. Chrysotherapy in rheumatoid arthritis with particular emphasis on the; effect of chrysotherapy on radiographical changes and on the optimal time of initiation of therapy. Scand J heumatol [Suppl] 1980; 34: 26-8.

[4] Dick W C, Deodhar S D, Provan C J, Nuki G, Buchanan W W.Isotope studies in normal and diseased joints: 99m-Tc uptake related to clinical assessment and synovial perfusion method by the 133-Xenon clearance technique. Clin Sci 1971; 40: 327-36.

[5] Oka M, Mottonen T, Rekonen A. Clinical results in the evaluation of peripheral joints by radionuclide methods. In:Kolarz G, Thumb N, eds. Methods of nuclear medicine in rheumatology. Stuttgart: Sqhattauer, 1982: 67-88.

[6] J. Christopher Buckland-Wright, Diana G. Macfarlane, Ignac Fogelman, et al.; Techetium 99m - methylene diphosphonate bone scanning in osteoarthritis hands, Nuclear Medicine and Molecular imaging Gateway(Springer), January 1991, Volume 18, Issue 1, pp 12-16.

[7] Bywaters E G L. Curven M, Dresner E, Dixon A. Ten-year follow-up of rheumatoid arthritis. Lancet 1960; ii: 1381.

[8] Teitsson I, Withrington R, Seifert M, Valdimarsson H.Prospective study of early rheumatoid arthritis. I. Prognostic value of IgA rheumatoid factor. Ann Rheum Dis 1984; 43:673-8.

[9] Duthie J J R, Brown P E, Knox J D E, Thompson M. Course and prognosis in rheumatoid arthritis. Ann Rheum Dis 1957; 16: 41123.

[10] Fleming A, Crown J M, Corbett M. Prognostic value of early features in rheumatoid disease. Br Med J 1976; i: 1243-5.

[11] Amos R 5, Constable T J, Crockson R A, Crockson A P,McConkey B. Rheumatoid arthritis: relation of serum Creactive protein and erythrocyte sedimentation rates to radiographic changes. Br Med J 1977; i: 195-7.

[12] Ochi T, Yonemasu K, Iwase R, Sasaki T, Tsuyama K, Ono K.Serum Clq levels as a prognostic guide to articular erosions in patients with rheumatoid arthritis. Arthritis Rheum 1984; 27:883-7.

[13] Westedt M-L, Daha M R, Baldwin W M, Stijnen T, Cats A.Serum immune complexes containing IgA appear to predict erosive arthritis in a longitudinal study in rheumatoid arthritis.Ann Rheum Dis 1986; 45: 809-15.

[14] Karan Peepre, Deepak Shah, AN Tiwari, et al. Clinical utility of nuclear medicine 99mTc-MDP bone scans in the early diagnosis of rheumatoid arthritis. Turk J Pediatr. 2008; 50:194-195. 